\title{
A Ratiometric Fluorescent Aptamer Homogeneous Biosensor Based on Hairpin Structure Aptamer for AFB1 Detection
}

\section{Beibei Feng}

Henan University of Technology

Fei Zhao

Henan University of Technology

Min Wei

Henan University of Technology

Yong Liu

Henan University

Xinyu Ren

Henan University of Technology

Zhiguang Suo ( $\square$ zg_suo@163.com )

Henan University of Technology https://orcid.org/0000-0001-8148-7778

\section{Research Article}

Keywords: ratiometric fluorescent, apt with hairpin structure, homogeneous sensor, aflatoxin B1 (AFB1)

Posted Date: January 3rd, 2022

DOI: https://doi.org/10.21203/rs.3.rs-1136909/v1

License: (9) (i) This work is licensed under a Creative Commons Attribution 4.0 International License. Read Full License 


\section{Abstract}

On the basis of aptamer (Apt) with hairpin structure and fluorescence resonance energy transfer (FRET), a ratio fluorescent aptamer homogeneous sensor was prepared for the determination of Aflatoxin $B_{1}$ (AFB1). Initially, the Apt labeled simultaneously with Cy5, BHQ2, and cDNA labeled with Cy3 were formed a double-stranded DNA through complementary base pairing. The fluorescent aptamer sensor demonstrates a weak fluorescence emission of Сy3 and a high fluorescence emission of Cy 5 due to the quenching effect of BHQ2. The double-stranded DNA structure will be disintegrated in the presence of AFB1, resulting the removal of Cy3 and the close of Cy 5 with BHQ2. The fluorescence signal of Cy3 and Cy5 were restored and quenched respectively. Thus, the ratio change of $\mathrm{F}_{\mathrm{Cy} 3}$ to $\mathrm{F}_{\mathrm{Cy} 5}$ was used to realized the detection of AFB1 with wider detection range and lower limit of detection (LOD). The response of the optimized protocol for AFB1 detection was wider linear range from $0.05 \mathrm{ng} / \mathrm{mL}$ to $100 \mathrm{ng} / \mathrm{mL}$ and the LOD was $12.6 \mathrm{pg} / \mathrm{mL}$. The sensor designed in this strategy has the advantages of simple preparation and fast signal response. It has been used for the detection of AFB1 in labeled corn and wine, indicating it had good application potential in practical samples.

\section{Introduction}

According to the statistics, about $25 \%$ of the agricultural products are contaminated with mycotoxins during crop growth, storage or processing in the world ${ }^{[1]}$. Fungal contamination of food is a global concern, most of the toxic substances produced by pollution have strong carcinogenic effects on human beings ${ }^{[2]}$. Aflatoxin is the most important and toxic mycotoxin among many mycotoxins and AFB1 is the most toxic aflatoxin. Many crops such as peanuts, corn and grains are vulnerable to AFB1 contamination, which makes AFB1 a serious threat to food safety and human health ${ }^{[3]}$. At present, more than 100 countries and regions have set limits for AFB1 in various foods ranging from 1 to $20 \mu \mathrm{g} / \mathrm{kg}{ }^{[4]}$. Therefore, there is an urgent need to establish a fast, safe, accurate and capable of on-site detection of AFB1 method.

At present, the methods used for AFB1 detection mainly include thin layer chromatography (LC), highperformance liquid chromatography (HPLC), high performance liquid chromatography-mass spectrometry (HPLC-MS) ${ }^{[4]}$. Although these methods have good accuracy and high sensitivity, they have disadvantages such as tedious sample pretreatment process, expensive instruments, highly trained professionals and so on, which limit their application for rapid and on-site screening of AFB1 ${ }^{[5]}$. Due to these disadvantages of traditional methods, fluorescence detection which has the advantages of high sensitivity, simple operation and simultaneous detection of multiple toxins, has attracted the attention of researcher ${ }^{[6]}$. With the rapid development in recent decades, various fluorescent biosensors have emerged in an endless stream ${ }^{[7]}$. In order to enhance the specific recognition of the sensor and the target, aptamer as target recognition element compared with antibody, enzyme, etc. 
Aptamer has stronger affinity with the target, the simpler and cheaper synthesis process and better stability. What's more, the aptamer is not easy to be limited by the storage environment, temperature, and its resistance to degeneration ${ }^{[8]}$. Li et al. reported a new aptamer fluorescence sensor, whcih can sensitively detect $\mathrm{Pb}^{2+}[9]$. Yang et al designed a novel fluorescence based protein detection method using aptamer probe as recognition molecule and cationic conjugated polymer (CCP) as reporter molecule ${ }^{[10]}$. At present, the fluorescence aptasensors prepared by using aptamer instead of antibody has been widely used in the detection of mycotoxins, protein, cell ${ }^{[11]}$.

Most of the fluorescence aptasensors require a tedious and time-consuming process of embedding the probe or some recognition molecules on the surface of the material used to prepare the sensor, which will greatly increase the preparation time of the sensor. In addition, the separation and purification process involved in fixing the probe and identifying the molecules will lead to the signal damage of the sensor and affect the performance of the sensor. To solve this problem, many researchers have chosen to use homogeneous sensors ${ }^{[12-13]}$, which is kept in a solution from preparation to detection without any separation operation. Fluorescence resonance energy transfer (FRET) is a commonly used method for quenching fluorescent dyes in fluorescence sensors. The FRET process requires a distance between the quenching material and the fluorescence probe within $10 \mathrm{~nm}$. When the quenching material and the fluorescence probe are dispersed in the system, the FRET process is weak. It almost has no effect on the signal of fluorescent probe, which is convenient for the application of homogeneous detection in fluorescent adaptor sensor. $\mathrm{Lu}^{[14]}$ et al. provided a simple, homogeneous and sensitive strategy mediated FRET for the histone analysis. The homogeneous sensor has been widely used in the detection of mycotoxins, protein, cell[ ${ }^{[15]}$.

Furthermore, the conventional fluorescent aptasensors were designed based on the changes of the "signal on" or "signal off" of single probe. The conventional fluorescent aptasensors were easily affected by the environmental and instrument factors in detection of the actual sample, resulting in the degradation of the sensor performances ${ }^{[16]}$. Yang et al. ${ }^{[17]}$ prepared a simple and rapid fluorescence homogeneous sensor for AFB1 detection by using the adaptation system of BHQ2-labeled complementary chain and Cy3 labeled fluorescent probe. However, the signal ratio is not sensitive to changes affected by environmental and instrumental. To improve the stability and accuracy of the sensor, the ratiometric fluorescent aptasensor was selects two signal probes with different excitation and emission wavelengths. The final signals are determined by the fluorescence signal ratio of the two probes [18-19]. Therefore, the ratiometric fluorescent aptasensor using two signal probes has become a new research hotspot.

In this paper, a simple, rapid and sensitive ratio homogeneous fluorescent aptasensor was constructed by using aptamer with hairpin structure labeled simultaneously with Cy5, BHQ2 and cDNA labeled with Cy3 to detect AFB1. The AFB1 aptamer with hairpin structure was used as recognition element, and the labeled BHQ2 whcih can quench the fluorescent dyes Cy3 and Cy5. The aptamer labeled with Cy5 and BHQ2 was paired with the cDNA labeled with Cy3 to form a double strand through complementary 
pairing. At this time, Cy3 was close to BHQ2, leading to fluorescence quenching. A high fluorescence emission of Cy5 emerged due to the distance of BHQ2. The hairpin structure would be opened in the presence of AFB1.The fluorescence intensity of Cy3 was increased due to it was far away from BHQ2, and the fluorescence intensity of Cy 5 was decreased due to Cy 5 approaching BHQ2. AFB1 was detected by comparing the ratio of $\mathrm{F}_{\mathrm{Cy} 3}$ to $\mathrm{F}_{\mathrm{Cy} 5}$ before and after adding AFB1.

\section{Materials And Methods \\ 2.1. Materials}

The AFB1 aptamer (Apt) and complementary DNA (cDNA) were synthesized by Sangon Biotechnology Co., Ltd (Shanghai, China). The sequences of the oligonucleotides are as follows: Apt: 5'-BHQ 2 -TGC ACG TGT TGT CTC TCT GTG TCT CG TGC-Cy5-3', cDNA: 5'-GCA CGT CTG GAC ACG AGA CAA CAC GTG CACy3-3'. AFB1, ochratoxin $A(O T A)$, fumonisin $B_{1}\left(F B_{1}\right)$, deoxynivalenol (DON), and zaralenone (ZEN) were purchased from Sigma Co., Ltd (USA). Sodium chloride ( $\mathrm{NaCl})$, Magnesium chloride $\left(\mathrm{MgCl}_{2}\right)$, Ethanol absolute $\left(\mathrm{C}_{2} \mathrm{H}_{6} \mathrm{O}\right)$ and hydrochloric acid $(\mathrm{HCl})$ were purchased from Kermel Chemical Reagent Co., Ltd (Tianjin, China). Tris (hydroxymethyl) aminomtethane was purchased from Shanghai Macklin Biochemical Co., Ltd (China). Ethylene diamine tetraacetic acid disodium salt (EDTA) was purchased from Luoyang Chemical Reagent Factory (Henan, China). $50 \mathrm{mM}$ Tris-HCl buffer (pH 7.4, $50 \mathrm{mM}$ Tris, 0.2 $\mathrm{M} \mathrm{NaCl}$ and $1 \mathrm{mM}$ EDTA) were used in this paper. All oligonucleotides were diluted with $50 \mathrm{mM}$ Tris- $\mathrm{HCl}$ buffer, and all reagents shall be fully mixed before use. All the water used in the experiment was sterilized ultra-pure water (18.2 M $\Omega$ ).

\subsection{Preparation of Cy5-Apt-BHQ2/cDNA-Cy3 sensor}

$20 \mu \mathrm{L}$ cDNA $(1 \mu \mathrm{M})$ was mixed with $18 \mu \mathrm{L}$ Apt $(1 \mu \mathrm{M})$ with stirring, the mixture was heated to $95^{\circ} \mathrm{C}$ in PCR and kept for 5 minutes, then slowly cooled to $4{ }^{\circ} \mathrm{C}$, Cy5-apt-BHQ2 / cDNA-Cy3 sensor was prepared and stored at $4{ }^{\circ} \mathrm{C}$ until use.

\subsection{Detection of AFB1}

$10 \mu \mathrm{L}$ AFB1 $(200 \mathrm{ng} / \mathrm{mL})$ was mixed with $38 \mu \mathrm{L}$ of prepared Cy5-Apt-BHQ2/cDNA- Cy3 sensor solution, and adding $20 \mathrm{mmol} / \mathrm{L}$ Tris- $\mathrm{HCl}(\mathrm{pH} 7.4)$ buffer solution to $200 \mu \mathrm{L}$. Then the mixed solution was incubated at $37{ }^{\circ} \mathrm{C}$ with a water bath thermostatic oscillator for 40 minutes. In this paper, excitation wavelength scanning method was used to detect fluorescence intensity. The fluorescence intensity was measured at the excitation wavelength scanning mode by Hitachi F-7100 fluorescence spectrophotometer (Japan). All measurements were all carried out at room temperature. The fluorescence cell was a quartz fluorescence cell with a slit of $2 \mathrm{~mm}$, and the emission spectrum of Cy3 probe was recorded in the excitation wavelength of $530 \mathrm{~nm}$ and in the wavelength range of $550 \mathrm{~nm}-650 \mathrm{~nm}$. The emission spectra of the Cy5 probe were recorded in the excitation wavelength of $635 \mathrm{~nm}$ and the wavelength range of $650 \mathrm{~nm}-750 \mathrm{~nm}$. The scanning voltage was $950 \mathrm{~V}$, and the excitation and emission slit widths were set to $5 \mathrm{~nm}$. All measurements were made at room temperature. Fluorescence 
spectrophotometer was used to record the fluorescence spectra of mixed solution before and after adding AFB1 at excitation wavelength of $530 \mathrm{~nm}$ (Cy3) and $635 \mathrm{~nm}$ (Cy5), the ratio value of fluorescence intensity of $\mathrm{Cy} 3\left(\mathrm{~F}_{\mathrm{Cy} 3}\right)$ to fluorescence intensity of $\mathrm{Cy} 5\left(\mathrm{~F}_{\mathrm{Cy} 5}\right)$ was compared before and after AFB1 added.All the test data were performed three times.

\subsection{Application of the fluorescence sensor in actual samples}

To study the feasibility of the designed sensor, the spiked corn flour samples were tested. The processing method of corn flour referred to Seok's ${ }^{[18]}$ processing method, $1 \mathrm{~g}$ corn meal sample was put into a 10 $\mathrm{mL}$ flask, and adding $1 \mathrm{~mL}$ analytically pure methanol was mixed with AFB1 in different known concentrations, eventually. The mixture was left in a fume hood for 12 hours to completely evaporate the solvent, then extracted at room temperature with methanol/water $(2: 8, \mathrm{v} / \mathrm{v})$ for 15 minutes. After centrifugation at $10000 \mathrm{rpm}$ for $15 \mathrm{~min}$, the extract was filtered through a $0.45 \mathrm{~mm}$ injection filter. The filtrate was diluted with $50 \mathrm{mM}$ Tris $-\mathrm{HCl}(\mathrm{pH} 7.4)$ to the concentration of $0.1 \mathrm{ng} / \mathrm{mL}, 1 \mathrm{ng} / \mathrm{mL}$, and 10 $\mathrm{ng} / \mathrm{mL}$. The prepared sensor was used to detect the spiked samples, and the fluorescence spectra of Cy3 and Cy 5 were recorded after the addition of the fluorescence sensor to the samples.

After removing the foam from the wine samples by nitrogen blowing, the spiked wine samples were detected. The toxin concentration was diluted with Tris- $\mathrm{HCl}(\mathrm{pH} 7.4)$ until $0.1 \mathrm{ng} / \mathrm{mL}, 1 \mathrm{ng} / \mathrm{mL}$ and 10 $\mathrm{ng} / \mathrm{mL}$ with different known concentrations of AFB1. By recording the fluorescence spectra of Cy3 and Cy5 of the fluorescence sensors after adding samples, the labeled samples were detected. All the test data were performed three times

The contaminated feed (corn peanut meal) was ground to powder $(1.0 \mathrm{~g})$ and the extraction solvent was added after stirring for $30 \mathrm{~min}$. The supernatant was treated to detect AFB1. All results were compared by HPLC. All the test data were performed three times

\section{Results And Discussion}

\subsection{The design principle of the aptasensor for AFB1 detection}

Ratiometric homogeneous fluorescent aptamer based on the hairpin structure of Apt and the characteristic that $\mathrm{BHQ} 2$ can simultaneously quench the fluorescence probe Cy3 and Cy5, a fluorescence aptamer homogeneous sensor was designed for the detection of AFB1. The detection principle was illustrated in Figure 1. The Apt labeled with Cy5 and BHQ2 and cDNA labeled with Cy3 form a doublestranded DNA through complementary base pairing. At this moment, Cy3 was close to BHQ2, resulting in fluorescence quenching. Due to the opening of the hairpin structure, Cy5 was far away from BHQ2 leads to the recovery of the fluorescence signal. The double-stranded DNA structure will be disintegrated in the presence of AFB1, resulting the removal of Cy3 and the close of Cy5 with BHQ2. At this time, a weak 
fluorescence emission of Cy3 and a high fluorescence emission of Cy5 due to the quenching effect of $\mathrm{BHQ}$ 2.By comparing the ratio change of $\mathrm{F}_{\mathrm{Cy} 3}$ to $\mathrm{F}_{\mathrm{Cy} 5}$ before and after adding AFB1, the detection of AFB1 was realized. Therefore, the ratiometric fluorescent aptamer homogeneous sensor can be used for AFB1 detection theoretically.

\subsection{The feasibility of the ratiometric fluorescent aptamer homogeneous sensor}

The feasibility of the prepared Cy5-Apt-BHQ2/cDNA-Cy3 sensor was analyzed in the absence and presence of AFB1. The result was shown in Figure. 2. The initial fluorescence intensity of the sensor showed that the ratio of $\mathrm{F}_{\mathrm{Cy} 3} / \mathrm{F}_{\mathrm{Cy} 5}$ was 1.1 in the absence of AFB1 (Red line). After adding $10 \mathrm{ng} / \mathrm{mL}$ AFB1 for incubation for 40 minutes, the value of the $F_{\mathrm{Cy} 3} / F_{\mathrm{Cy} 5}$ was increased 6 times compared to without AFB1, indicating the feasibility of the prepared sensor for AFB1 detection (Blue line). The doublestranded DNA structure will be disintegrated in the presence of AFB1, resulting the removal of Cy3 and the close of Cy5 with BHQ2. Due to the fluorescence quenching effect of $\mathrm{BHQ} 2$, the ratio of $\mathrm{F}_{\mathrm{Cy} 3} / \mathrm{F}_{\mathrm{Cy} 5}$ increased to 6.6 when adding $10 \mathrm{ng} / \mathrm{mL}$ AFB1. The results were shown that the ratiometric fluorescent aptamer homogeneous sensor can be used to detect AFB1, sensitively.

\subsection{Optimization of the experiment parameter}

In order to get the optimal performance of the prepared sensor, the ratio value between Apt and cDNA should be optimized. The background signal will be affect by the change of the ratio value Apt to cDNA Because changing the ratio value of Apt to cDNA would affect the ratio of the initial signal $\mathrm{F}_{\mathrm{Cy} 3}$ to $\mathrm{F}_{\mathrm{Cy} 5}\left[\left(\mathrm{~F}_{\mathrm{Cy} 3} / \mathrm{F}_{\mathrm{Cy} 5}\right)_{0}\right],\left(\mathrm{F}_{\mathrm{Cy} 3} / \mathrm{F}_{\mathrm{Cy} 5} /\left(\mathrm{F}_{\mathrm{Cy} 3} / \mathrm{F}_{\mathrm{Cy} 5}\right)_{0}\right.$ was used to reflect the optimization results ${ }^{[18]}$. As shown in Figure. 3, $\left(\mathrm{F}_{\mathrm{cy} 3} / \mathrm{F}_{\mathrm{cy} 5}\right) /\left(\mathrm{F}_{\mathrm{cy} 3} / \mathrm{Fc}_{\mathrm{y} 5}\right)_{0}$ was growing with the increasing the proportion between Apt and cDNA from 0.7 to 0.9. When the proportion between Apt and cDNA was greater than 0.9, $\left(\mathrm{F}_{\mathrm{cy} 3} / \mathrm{F}_{\mathrm{cy} 5}\right) /\left(\mathrm{F}_{\mathrm{cy} 3} / \mathrm{F}_{\mathrm{cy} 5}\right)_{0}$ started to decrease. This was because the double-strand DNA formed by Apt and cDNA has reached a saturation state, and the redundant Apt reduces the sensitivity of the sensor and the signal change was no longer obvious. Therefore, 0.9 was selected as the optimal ratio value between Apt and cDNA.

\subsection{Detection of AFB1}

Under the optimal experimental parameters, $\mathrm{F}_{\mathrm{Cy} 3} / \mathrm{F}_{\mathrm{Cy} 5}$ was measured by analyzing $\mathrm{AFB} 1$ solution with different concentrations. The detected spectrogram was shown in Figure. 4(A), the fluorescence intensity of the fluorescence sensor Cy3 $\left(\mathrm{F}_{\mathrm{cy} 3}\right)$ increased and the fluorescence intensity of Cy5 $\left(\mathrm{F}_{\mathrm{cy} 5}\right)$ decreased with the increasing concentration of AFB1 added $(0,0.05,0.1,0.5,1,10,50,100,500 \mathrm{ng} / \mathrm{mL})$. A good linear relationship was obtained between $0.05 \mathrm{ng} / \mathrm{mL}$ and $100 \mathrm{ng} / \mathrm{mL}$ (Figure. 4B), the linear equation was $\frac{F_{C y 3}}{F_{C y 5}}=2.34 L g C_{A F B 1}+4.60\left(R^{2}=0.994\right)$

The LOD was calculated to be $12.6 \mathrm{pg} / \mathrm{mL}$ ( $3 \sigma$ rule). The result indicated the prepared ratiometric homogeneous fluorescent aptasensor had a wider linear range and lower detection limit for AFB1 detection. 
In order to study the specificity of the prepared fluorescence aptasensor to different toxins. Under the same experimental condition, $10 \mathrm{ng} / \mathrm{mL}$ of $A F B 1,100 \mathrm{ng} / \mathrm{mL}$ of interference toxins such as OTA, FB1, DON, ZEN and mixture of AFB1 with all interference toxins were detected and results were shown in Figure. 5. After incubating the prepared aptasensor with a single interfering toxin for 40 minutes, the ratio value of fluorescence intensity of the two dyes $\left(\mathrm{F}_{\mathrm{Cy} 3} / \mathrm{F}_{\mathrm{Cy} 5}\right)$, remained unchanged even though its concentration was 10 times higher than that of AFB1. However, as long as AFB1 was present, the value of $\mathrm{F}_{\mathrm{Cy} 3} / \mathrm{F}_{\mathrm{Cy} 5}$ of fluorescence intensity could be significantly increased after incubation for detection, whether AFB1 was existed alone or AFB1 was mixed with interfering toxins, indicating that the designed aptasensor had good specificity.

The detection of the designed aptasensor was compared with other AFB1 aptasensors, and the results are shown in Table 1. It can be seen that the aptasensor designed in this experiment had a good linear range and a lower LOD compared with most sensors. This result indicated that the dual-signal change of the ratio sensor can effectively improve the sensitivity of the fluorescence aptasensor, and the homogeneous detection method improved the stability of the sensor.

Table 1

Comparison of other sensors developed for AFB1 detection

\begin{tabular}{|llll|}
\hline Methods & Linear range $(\mathrm{ng} / \mathrm{mL})$ & LOD $(\mathrm{ng} / \mathrm{mL})$ & Reference \\
\hline Electrochemistry & $0.6-313$ & 0.6 & {$[19]$} \\
\hline ELISA & $0.24-2.21$ & 0.13 & {$[20]$} \\
\hline Liquid Chromatography & $0.05-2$ & 0.108 & {$[21]$} \\
\hline Electrochemistry & $0.05-20$ & 0.016 & {$[22]$} \\
\hline Fluorescence & $0.1-0.8$ & 0.07 & {$[23]$} \\
\hline Fluorescence & $0.05-100$ & 0.05 & {$[24]$} \\
\hline Fluorescence & $0.015-15$ & 0.003 & {$[25]$} \\
\hline Fluorescence & $0.05-100$ & 0.0126 & This work \\
\hline
\end{tabular}

\subsection{Detection of the actual samples}

In order to further verify the accuracy and practicability of the ratio homogeneous fluorescent apatasensor, the spiked corn flour samples and spiked wine samples were tested, and the scalars were $0.1 \mathrm{ng} / \mathrm{mL}, 1 \mathrm{ng} / \mathrm{mL}$, and $10 \mathrm{ng} / \mathrm{mL}$, respectively. The detection results were shown in Table 2 . The recovery of the detection results were $95-110 \%$ and $93-108 \%$, respectively. The relative standard deviation (RSD) were not more than $10 \%$. HPLC and the prepared fluorescence sensor were used to detect the 
contaminated feed. The results showed that the prepared sensor had certain accuracy and practicability which can be used as an alternative to traditional detection methods.

Table 2

Detection of AFB1 in corn meal and wine samples $(n=3)$

\begin{tabular}{|llllll|}
\hline Sample & $\begin{array}{l}\text { Spiked amount } \\
(\mathrm{ng} / \mathrm{mL})\end{array}$ & $\begin{array}{l}\text { Measured by HPLC } \\
(\mathrm{ng} / \mathrm{mL})\end{array}$ & $\begin{array}{l}\text { Found amount } \\
(\mathrm{ng} / \mathrm{mL})\end{array}$ & $\begin{array}{l}\text { Recovery } \\
(\%)\end{array}$ & $\begin{array}{l}\text { RSD } \\
(\%)\end{array}$ \\
\hline Corn & 0 & $\mathrm{ND}$ & $\mathrm{ND}$ & - & - \\
\cline { 2 - 6 } & 0.1 & 0.09 & 0.11 & 110 & 9.3 \\
\hline 1 & 1.1 & 0.95 & 95 & 7.1 \\
\hline wine & 0 & 10.47 & 9.56 & 96 & 6.9 \\
\cline { 2 - 6 } & 0.1 & $\mathrm{ND}$ & $\mathrm{ND}$ & - & - \\
\cline { 2 - 6 } & 1 & 0.097 & 0.093 & 93 & 8.2 \\
\hline ND: Not detected. & 1.03 & 0.98 & 98 & 6.2 \\
\hline
\end{tabular}

\section{Conclusions}

In summary, combining Apt with hairpin structure labeled with Cy5 and BHQ2 and cDNA labeled with Cy3 to detect AFB1, a simple, rapid and sensitive ratio homogenous fluorescent aptasensor was constructed. The ratio value of fluorescence intensity between Cy3 and Cy $5\left(\mathrm{~F}_{\mathrm{Cy} 3} / \mathrm{F}_{\mathrm{Cy} 5}\right)$, and $\mathrm{LgC}_{\mathrm{AFB} 1}$ showed a good linear relationship within the concentration range of AFB1 from $0.05 \mathrm{ng} / \mathrm{mL}$ to $100 \mathrm{ng} / \mathrm{mL}$, and the LOD was $12.6 \mathrm{pg} / \mathrm{mL}$. The recovery rates of AFB1 in spiked corn meal samples and wine were $92-110 \%$ and $88-112 \%$, respectively. Furthermore, the adequate determination of AFB1 in corn, wine samples designed in this paper confirmed the applicability of this strategy compared with HPLC. The aptasensor designed in this paper is simple in preparation, fast in signal response, which has the application prospect of realizing fast detection of AFB1 on site.

\section{Declarations}

\section{Ethics Approval}

Not applicable.

\section{Consent to Participate}

Not applicable. 


\section{Consent for Publication}

Not applicable.

\section{Conflict of interest}

The authors have no relevant financial or non-financial interests to disclose.

\section{Funding}

This study was funded by the Key Scientific and Technological Project of Henan Province (212102310001) and Science Foundation of Henan University of Technology (31401425).

\section{Authors' Contributions}

Beibei Feng. concept design, editing, Review modification, editing, and analysis data. Fei Zhao: Performed the experiments and modification. Min Wei: Corresponding author, concept design, Review writing and editing. Yong Liu: Contributed reagents, materials. Xinyu Ren: Contributed reagents. Zhiguang Suo: Review modification, editing, and Analyzed and interpreted the data.

\section{Data Availability}

Not applicable.

\section{References}

1. Al-Taher F, Banaszewski K, Jackson L, Zweigenbaum J, Ryu D, Cappozzo J (2013) Rapid Method for the Determination of Multiple Mycotoxins in Wines and Beers by LC-MS/MS Using a Stable Isotope Dilution Assay. Food Chem 61(10):2378-84.

2. D. Pattono, P.F. Gallo, T. Civera (2011)Detection and quantification of Ochratoxin A in milk produced in organic farms. Food Chem 127 374-377.

3. S. Sun, R. Zhao, Y. Xie, Y. Liu (2019) Photocatalytic degradation of aflatoxin B-1 by activated carbon supported TiO2 catalyst. Food Control 100:183-188.

4. S.M. Sun, R. Zhao, Y.L. Xie, Y. Liu (2021) Reduction of aflatoxin B-1 by magnetic graphene oxide/TiO2 nanocomposite and its effect on quality of corn oil. Food Chem 343

5. B KAA, D NMDC, A MA, C MR, E ASE, F RZ (2017) A new amplified r-shape electrochemical aptasensor for ultrasensitive detection of aflatoxin B 1. Biosens. Bioelectron 94:374-379.

6. Wu S, Duan N, Zhu C, Ma X, Wang M, Wang Z (2011) Magnetic nanobead-based immunoassay for the simultaneous detection of aflatoxin B-1 and ochratoxin A using upconversion nanoparticles as multicolor labels. Biosens. Bioelectron 30(1):35-42.

7. Micheli L, Grecco R, Badea M, Moscone D, Palleschi G (2005) An electrochemical immunosensor for aflatoxin M1 determination in milk using screen-printed electrodes. Biosens. Bioelectron 21(4):588- 
96.

8. Li Q, Lu Z, Tan X, Xiao X, Wang P, Wu L (2017) Ultrasensitive detection of aflatoxin B-1 by SERS aptasensor based on exonuclease-assisted recycling amplification. Biosens. Bioelectron 97:59-64.

9. Chen M, Hassan M, Li H, Chen Q (2020) Fluorometric determination of lead(II) by using aptamerfunctionalized upconversion nanoparticles and magnetite-modified gold nanoparticles. Microchimica Acta: 187(1).

10. Yang XH, Wang SF, Wang KM, Luo XM, Tan WH, Cui L (2009) Novel Method of Protein Detection Based on Cationic Conjugated Polymer and Aptamer Probe. Chemical Chem Res Chin Univ 30(5):899-902.

11. Lu X, Jia H, Yan X, Wang J, Wang Y, Liu C (2018) Label-free detection of histone based on cationic conjugated polymer-mediated fluorescence resonance energy transfer. Talanta 180:150-5.

12. Chen J, Liu J, Chen X, Qiu H (2019) Recent progress in nanomaterial-enhanced fluorescence polarization/anisotropy sensors. Chin Chem Lett 30(9):1575-80.

13. Figureueroa-Miranda G, Feng L, Shiu SC-C, Dirkzwager RM, Cheung Y-W, Tanner JA (2018) Aptamerbased electrochemical biosensor for highly sensitive and selective malaria detection with adjustable dynamic response range and reusability. Sensors and Actuators B-Chemical 255:235-43.

14. Joo M, Baek SH, Cheon SA, Chun HS, Choi S-W, Park TJ (2017) Development of aflatoxin B-1 aptasensor based on wide-range fluorescence detection using graphene oxide quencher. Colloids Surf. B 154:27-32.

15. Yang Y, Li W, Shen P, Liu R, Li Y, Xu J (2017) Aptamer fluorescence signal recovery screening for multiplex mycotoxins in cereal samples based on photonic crystal microsphere suspension array. Sens. Actuators B Chem 248:351-8.

16. Huang J, Chen Y, Yang L, Zhu Z, Zhu G, Yang X (2011) Amplified detection of cocaine based on strand-displacement polymerization and fluorescence resonance energy transfer. Biosens. Bioelectron 28(1):450-3.

17. Seok Y, Byun J-Y, Shim W-B, Kim M-G (2015) A structure-switchable aptasensor for aflatoxin B1 detection based on assembly of an aptamer/split DNAzyme. Anal. Chim. Acta 886:182-7.

18. Kim D-H, Seong J, Lee H, Lee K-H (2014) Ratiometric fluorescence detection of Hg(II) in aqueous solutions at physiological $\mathrm{pH}$ and live cells with a chemosensor based on tyrosine. Sens. Actuators $\mathrm{B}$ Chem 196:421-8.

19. Figureueroa-Miranda G, Feng L, Shiu SC-C, Dirkzwager RM, Cheung Y-W, Tanner JA (2018) Aptamerbased electrochemical biosensor for highly sensitive and selective malaria detection with adjustable dynamic response range and reusability. Sens. Actuators B Chem 255:235-43.

20. Wang Y, Hu X, Pei Y, Sun Y, Wang F, Song C (2015) Selection of phage-displayed minotopes of ochratoxin A and its detection in cereal by ELISA. Anal. Methods 7(5):1849-54.

21. Aresta A, Vatinno R, Palmisano F, Zambonin CG (2006冈Determination of Ochratoxin A in wine at sub $\mathrm{ng} / \mathrm{mL}$ levels by solid-phase microextraction coupled to liquid chromatography with fluorescence detection. J. Chromatogr 1115(1-2):196-201. 
22. Li Y, Liu D, Zhu C, Shen X, Liu Y, You T (2020) Sensitivity programmable ratiometric electrochemical aptasensor based on signal engineering for the detection of aflatoxin B1 in peanut. J. Hazard. Mater: 387.

23. Guo M, Hou Q, Waterhouse GIN, Hou J, Ai S, Li X (2019) A simple aptamer-based fluorescent aflatoxin B1 sensor using humic acid as quencher. Talanta: 205.

24. Lu X, Wang C, Qian J, Ren C, An K, Wang K (2019) Target-driven switch-on fluorescence aptasensor for trace aflatoxin B1 determination based on highly fluorescent ternary CdZnTe quantum dots. Anal. Chim. Acta 1047:163-71.

25. Wang B, Zheng J, Ding A, Xu L, Chen J, Li CM (2019) Highly sensitive aflatoxin B1 sensor based on DNA-guided assembly of fluorescent probe and TdT-assisted DNA polymerization. Food Chem 294:19-26.

\section{Figures}

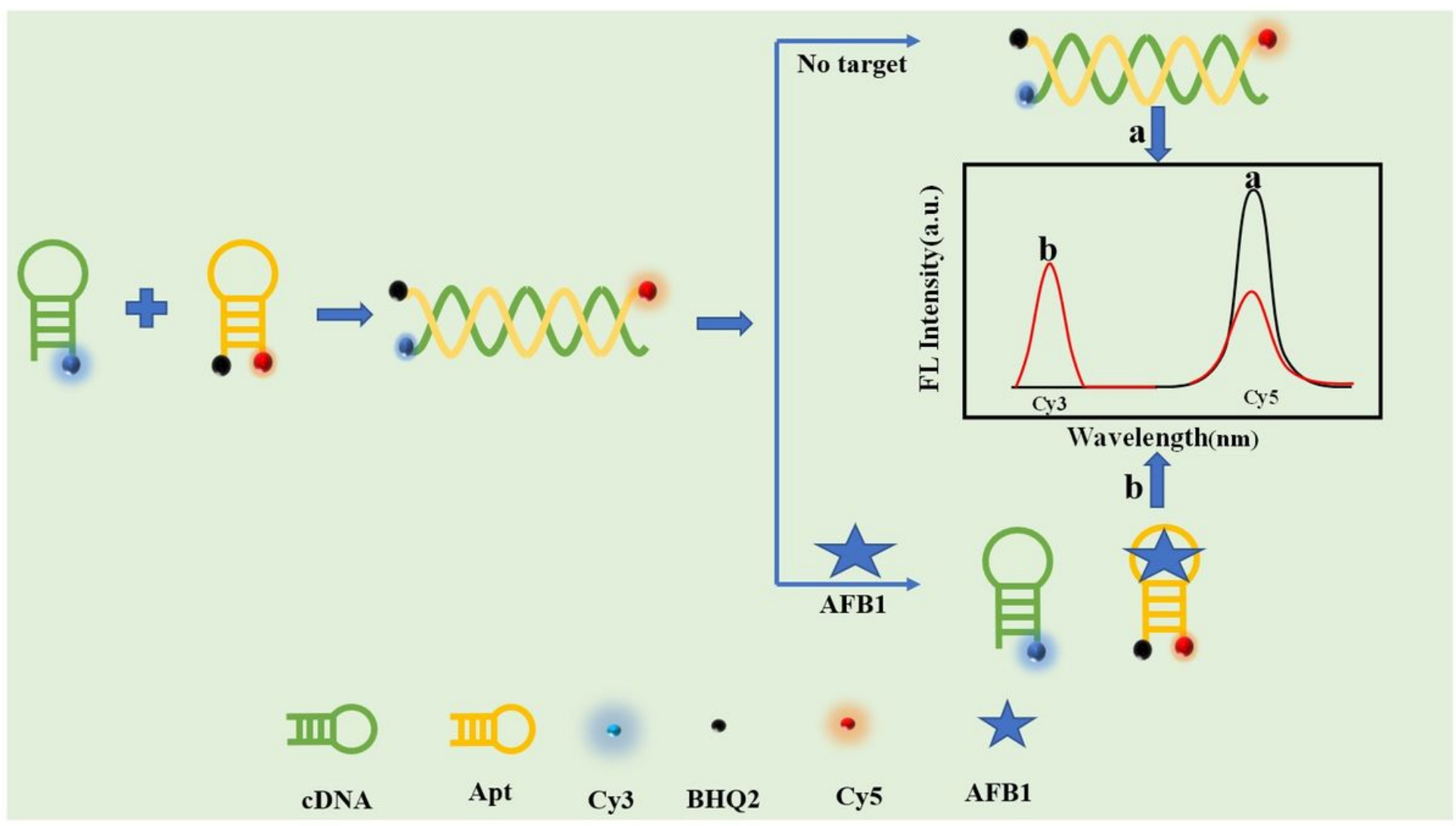

\section{Figure 1}

Preparation of Cy5-Apt-BHQ 2 / CDNA-Cy3 sensor and its detection principle diagram of AFB1 


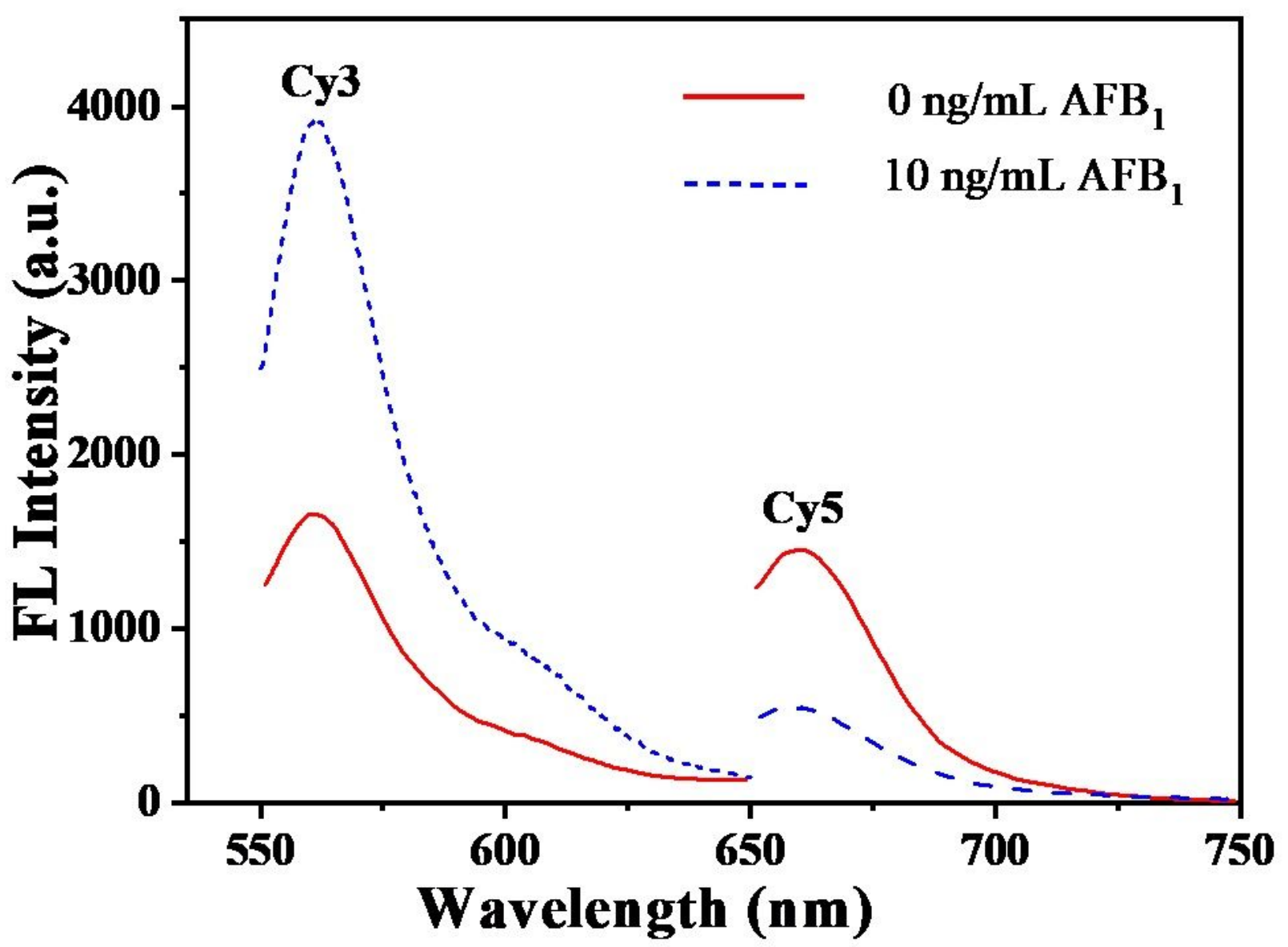

Figure 2

The fluorescence spectra of Cy5-Apt-BHQ2 / cDNA-Cy3 sensor before and after AFB1 incubation (The concentration of AFB1 is $10 \mathrm{ng} / \mathrm{mL}$ ) 


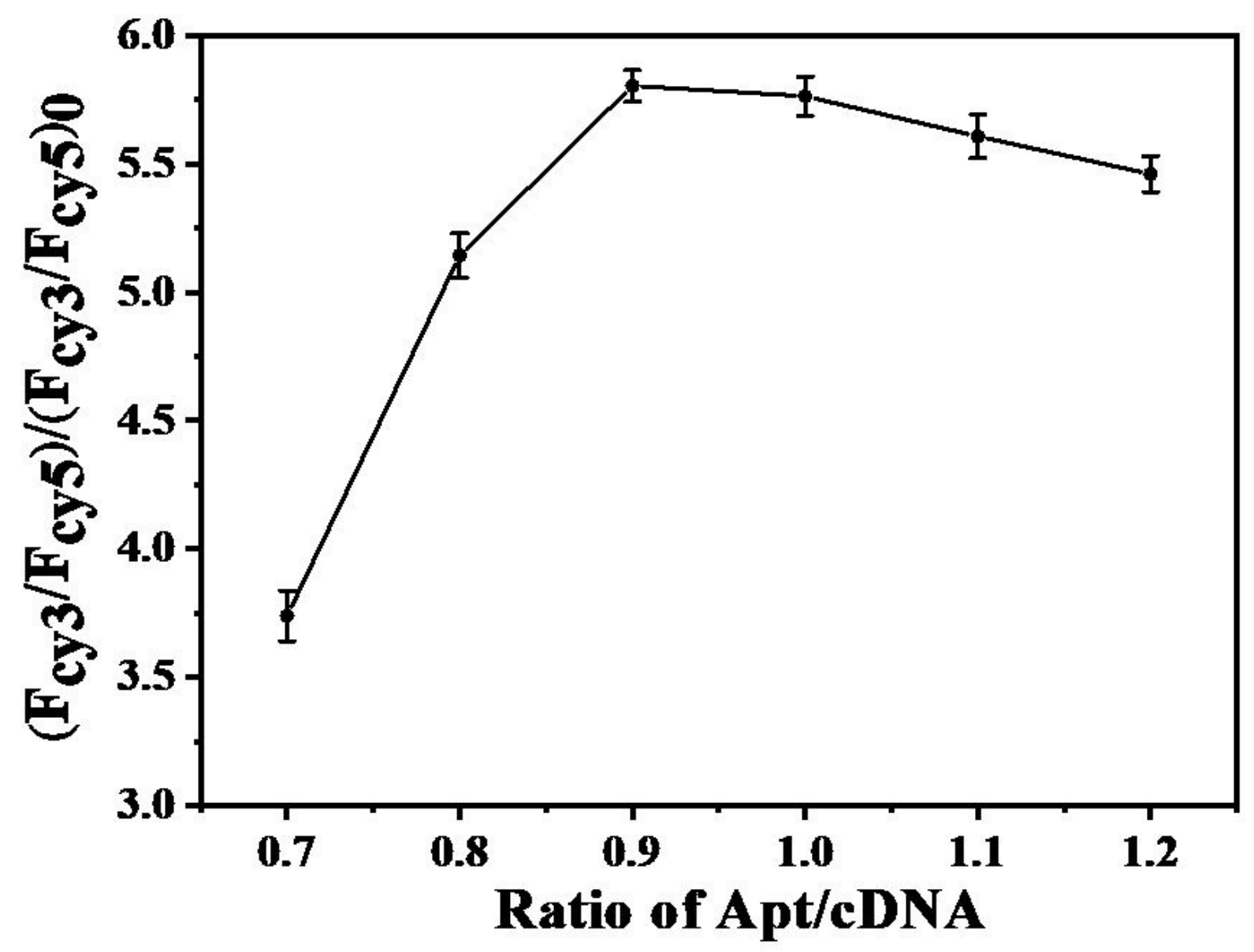

Figure 3

Effect of Apt and cDNA dosage ratio on Cy5-Apt-BHQ2 / cDNA-Cy3 sensor (AFB1 concentration is 10 $\mathrm{ng} / \mathrm{mL}$ )
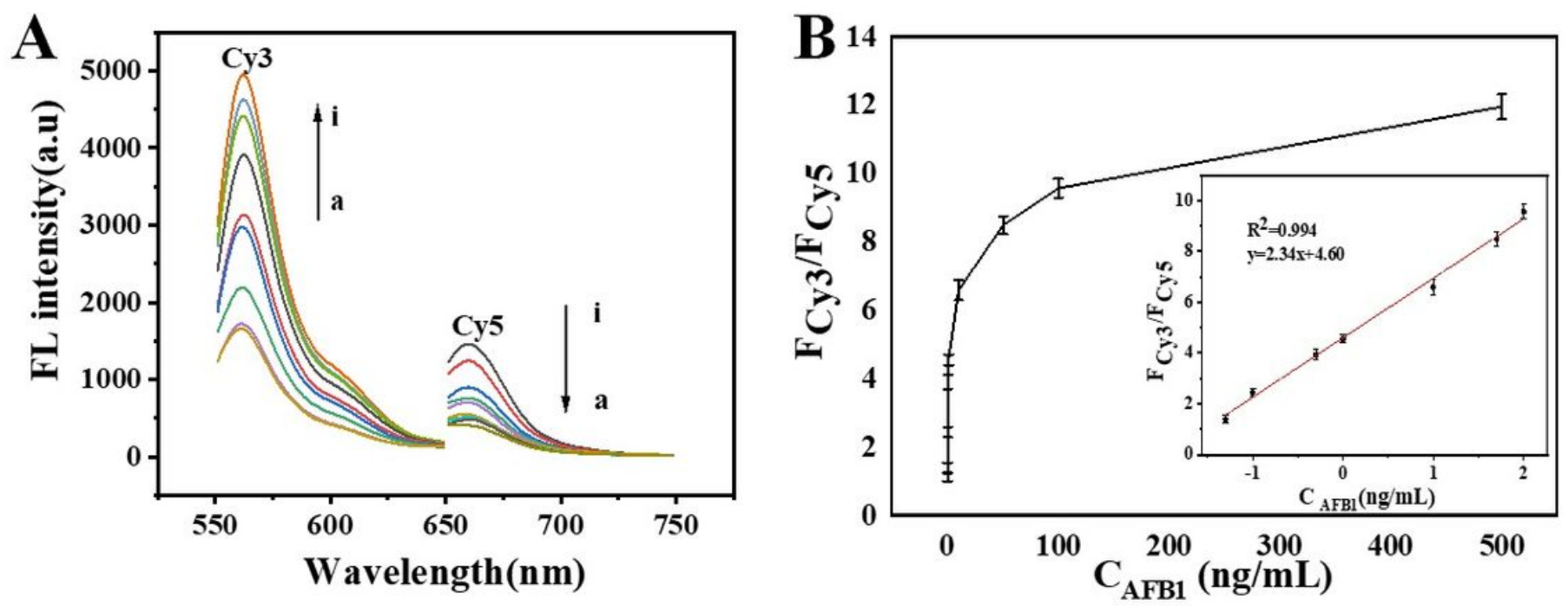
Figure 4

(A). Fluorescence spectra of AFB1 detected by Cy5-Apt-BHQ 2 / cDNA-Cy3 sensor in different concentration ranges. (a) $0 \mathrm{ng} / \mathrm{mL}$ (b) $0.05 \mathrm{ng} / \mathrm{mL} \otimes$ (c) $0.1 \mathrm{ng} / \mathrm{mL} \otimes$ (d) $0.5 \mathrm{ng} / \mathrm{mL} \rrbracket$ (e) $1 \mathrm{ng} / \mathrm{mL} \otimes$ (f) 10

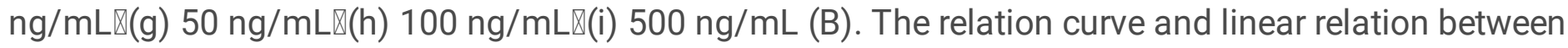
Fcy3/Fcy5 and AFB1 concentration.

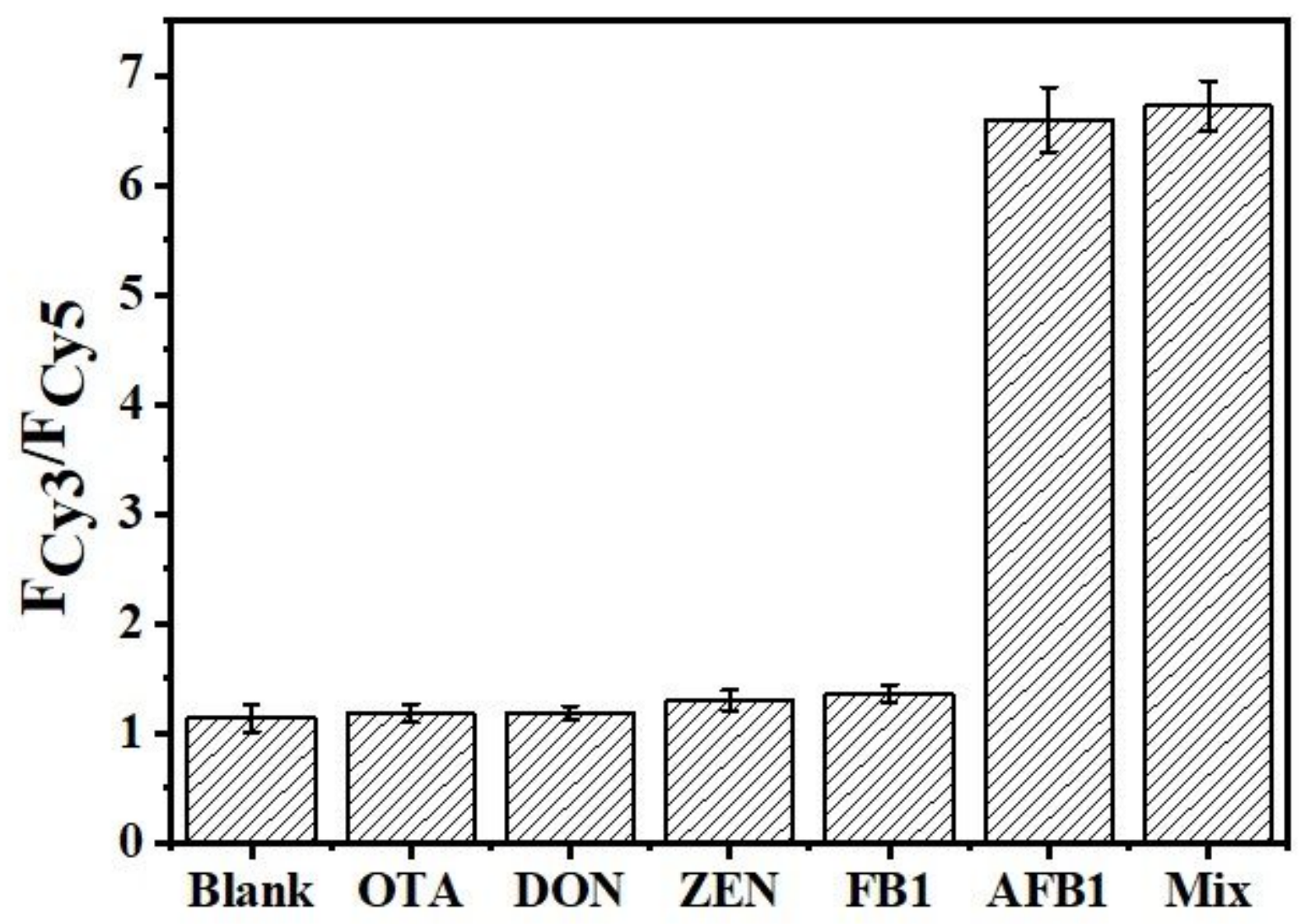

Figure 5

Histogram of the effects of different toxins on the fluorescence signal of Cy5-Apt-BHQ2 / cDNA-Cy3 sensor 